Saudi Journal of Oral and Dental Research

Abbreviated Key Title: Saudi J Oral Dent Res

ISSN 2518-1300 (Print) |ISSN 2518-1297 (Online)

Scholars Middle East Publishers, Dubai, United Arab Emirates

Journal homepage: https://saudijournals.com/sjodr

\title{
Impact of Alveolar Ridge Preservation on Implant Placement
}

\author{
Mohammed F Felemban ${ }^{1 *}$, Ji Li ${ }^{3}$, Fernando Suarez ${ }^{4}$, Edwin Sutherland ${ }^{2}$, Tapan N Koticha ${ }^{2}$
}

${ }^{1}$ Department of Dental Science, College of Dentistry, Taif University, Taif, Saudi Arabia

${ }^{2}$ Department of Periodontics, College of Dentistry, University of Oklahoma Health Science Center, Oklahoma City, Oklahoma, United State of America

${ }^{3}$ Department of Biostatistics, College of Public Health, University of Oklahoma Health Science Center, Oklahoma City, Oklahoma, United State of America

${ }^{4}$ Private Practice, Tacoma, Washington

DOI: $\underline{10.36348 / \text { sjodr.2020.v05i08.011 }}$

| Received: 18.08.2020 | Accepted: 26.08.2020 | Published: 30.08.2020

*Corresponding author: Mohammed F Felemban

\section{Abstract}

Aim: (a) to investigate the effect of Alveolar Ridge Preservation (ARP) on subsequent implant placement (IP) procedures and (b) to identify prognostic factors for ARP in relation to future IP. Material and Methods: Electronic and paper records of patients receiving one or more extractions with or without ARP followed by IP were reviewed in this study. Any sites with previously missing teeth, those that received immediate IP, growth factors, records with inadequate information or missing data were excluded. The need for additional grafting at IP, additional grafting prior to IP and sinus augmentation were the primary outcome measures. Statistical analyses were performed. Results: Out of a total of 1640 records reviewed, 829 patients with 1416 implants were included in the data analysis. There were no statistically significant differences between the sites that received ARP following extraction and the naturally healing sites on the need for grafting at IP, grafting prior to IP and sinus augmentation. The type of operator (general dentist or periodontist), smoking status, use of membrane at the time of ARP, loss of socket wall at the time of extraction and history for periodontal disease were confounding variables. Conclusions: Within the limitations of this study, ARP procedure used of implant site preparation did not show addition benefit on the future implant related outcome.

Keywords: alveolar socket, alveolar ridge preservation, dental implants, tooth extraction retrospective studies, alveolar ridge augmentation, sinus augmentation.

Copyright @ 2020: This is an open-access article distributed under the terms of the Creative Commons Attribution license which permits unrestricted use, distribution, and reproduction in any medium for non-commercial use (NonCommercial, or CC-BY-NC) provided the original author and source are credited.

\section{Clinical Relevance}

Scientific rationale for the study: Evidence from literature suggests that Alveolar Ridge Preservation procedures reduce dimensional changes in residual ridges following tooth extraction. The current study retrospectively evaluated if Alveolar Ridge Preservation resulted in improved implant placement outcomes without the need for additional bone augmentation in a large population of 829 patients with 1416 implants.

Principal findings: The need for grafting at the time of implant placement, grafting before implant placement and sinus augmentation group as implant related outcome revealed no differences between the sites that received alveolar ridge preservation procedure and the sites that received extraction only.

Practical implications: ARP may not have significant benefits on future implant placement. The effect may be greater in smokers or individuals with a history of periodontal disease and at sites that had a missing buccal plate at the time of extraction.

\section{INTRODUCTION}

The alveolar process is formed by the compact and cancellous bony structures that surrounds and support the teeth [1]. Following tooth extraction, the alveolar process undergoes significant structural changes resulting in horizontal and vertical dimensional loss [1-4]. While this phenomenon lacked of proper understanding and consideration for decades, multiple investigations have been conducted to evaluate the morphological changes of the alveolar crest following tooth extraction [5-9]. Similarly, dental implants have been the focus of numerous investigations since their introduction in 1970's. As a result of this extensive investigation, it is accepted today that dental implants are a prosthetic driven treatment modality and that the proper 3-dimensional positioning represents a factor of paramount importance $[10,11]$. Consequently, the 
volumetric changes and bone loss occurring following tooth extraction represent a phenomenon of key relevance [6].

In order to prevent or minimize the alveolar bone resorption that occurs following tooth extraction, the concept of alveolar ridge preservation (ARP) was introduced in 1982 by Ashman and Bruins [12, 13]. This procedure was named "Bone Maintenance" and the socket was filled with acrylic bone cements aiming at preserving the alveolar ridge. Since then, numerous investigations have been conducted to assess the efficacy ARP procedure [14-19]. Similarly, multiple systematic review and meta-analysis have been recently published in the last decade comparing the different types of grafting material used in ARP. Irrespective to the graft material, ARP have been proven to reduce the amount of horizontal and vertical bone loss in comparison to natural healing socket $[20,21,22]$. As such, Avila-Ortiz and collaborators found that ARP reduce the bucco-lingual bone loss by $1.89 \mathrm{~mm}$ and vertical bone height by $1.6 \mathrm{~mm}$ when compared to natural healing socket [23]. Differences have also been found histologically and histomorphometrically between the grafting materials [24, 25]. The highest percentage of vital bone was found in the socket grafted with alloplastic material, while the least was in the socket graft with xenograft. Allograft showed to be associated with more connective tissue formation and less graft particles after healing.

While the cascade of events occurring following tooth extraction continue to be further studied and elucidated, the clinical implications of grafted and natural healing sockets are still unclear. Today, the current evidence suggests that ARP might decrease the need for additional ridge augmentation during implant placement [20, 21, 26-28]. However, conflicting results have been reported with multiple systematic reviews concluding that there is not enough evidence to confirm that the employment of different socket grafting techniques would impact implant treatment outcome such as implant placement feasibility and need for further augmentation when compared to naturally healed site [26]. Therefore, it was recommended by the $4^{\text {th }}$ European Association for Osseointegration (EAO) consensus conference that more research is needed to investigate the relevance of ARP and implant related outcomes [27].

In 2015, a systematic review asked the question "Is there any additional benefit of ARP over unassisted healing in terms of the need for further augmentation" [26]. They concluded that while ARP could result in reduced need for augmentation at implant placement, naturally healed site did not prevent implant placement (IP). However, they also pointed out that IP and augmentation may have been influenced by surgical factors such as anatomic location, angulation of implant placement according to a prosthetically driven protocol or the diameter of the implants. With the current state of evidence, it is still not clear if ARP benefits future IP and how?

The purpose of this retrospective study is to investigate the influence of ARP procedures for future IP. Prognostic indicators for ARP in relation to subsequent IP were also evaluated.

\section{MATERIALS AND METHODS Study Design}

This study was approved by the Institutional Review Board at the University of Oklahoma Health Sciences Center. A chart review of all patients who received one or more dental implants in Graduate Periodontics (GP), Advance Educational in General Dentistry (AEGD), and Faculty Practice (FP) Clinics at University of Oklahoma Health Science Center (OUHSC) from 1 January 2000 - 31 December 2017 was done by one author (M.F.F.). Prospective records were identified using the electronic health record systems at the College of Dentistry, University of Oklahoma Health Sciences Center. Patients who received a tooth extraction followed by an implant placement were included. Exclusion criteria included the following: (I) site received immediate implant; (II) site received growth factor products at the time of extraction; (III) Records lacking adequate information and/or follow up data; (IV) Missing records.

\section{Clinical procedure}

All patients underwent tooth extraction with or without ARP followed by delayed IP. For extraction procedures: Local anesthesia was administered as needed at the extraction sites, tooth/teeth were removed as atraumatically as possible. Surgical curettes were used to degranulate the extraction socket. Following saline irrigation, some of the sites were left to heal naturally; the others were filled with bone graft and covered with collagen plugs or membrane. The extraction sites were secured with sutures to protect and prevent the dislodgment of the grafting materials. After the healing period, these sites were planned for IP. Full thickness flaps were reflected and the implant was placed per manufacturer recommended protocols. Ridge augmentation at the time of IP, ridge augmentation prior to IP or sinus augmentation prior to IP were performed as needed. Ridge augmentation prior to implant placement consisted of a separate surgical procedure aimed at increasing ridge width and/or height to allow placement of the implant in a prosthetically driven location later. Antibiotics and non-steroidal antiinflammatory (NSAIDs) medication were prescribed for 3-7 days following surgical procedures.

\section{Definition of Alveolar Ridge Preservation, Grafting at Implant Placement and Other Variables:}

ARP procedure was defined as extraction of specific tooth, filling the socket with grafting materials and covering the graft with a collagen plug or 
membrane. Naturally healing sockets were defined as sites where extractions were performed without the use of grafting materials or membrane. Post-operative complications were defined as unexpected bone loss after implant placement, the need for additional unexpected treatments at the first year following implant placement or early implant failure. Any implant that was removed before delivering the final restoration was considered as early implant failure. History of periodontal disease was defined as any patient diagnosed with periodontitis [29] or received active periodontal treatment (nonsurgical or surgical) at any time point prior to IP.

Implants were classified on the basis of diameter (regular diameter $>3.3 \mathrm{~mm}$ and narrow diameter $\leq 3.3 \mathrm{~mm}$ ) [30] and length (regular length $>8 \mathrm{~mm}$ and short length $\leq 8 \mathrm{~mm}$ ) [31].

\section{DATA EXTRACTION AND ANALYSIS}

All records included in the study after the initial assessment subjected to a manual data extraction process. This process was carried out by one author (MFF) based on the pre-identified format.

\section{Data was categorized under the following major headings}

- Primary Outcome Measures: Include Need for additional grafting at the time of implant placement recorded as (yes/no); Need for ridge augmentation prior to implant placement recorded as (yes/no); Need for sinus augmentation recorded as (yes/no).

- Operator Variables: Include the operator type (general dentist/periodontist) and the level of experience (faculty/resident).

- Complications: Include Intra-operative complications recorded as Complication/No Complication; Early implant failures recorded as (yes/no); and Post-operative complications including Bone Loss (yes/no) and need for further grafting (yes/no), need further soft tissue augmentation (yes/no).

- Patient demographic variable: Including selfreported age, gender, and race.

- Patient systemic variable: Diabetes Mellitus (DM) recorded as (yes/no); smoking status at the time of the procedure recorded as (yes/no); and intake of medications known to affect bone metabolism and/or dental implants recorded as (yes/no).

- Site - specific variable: Periodontal disease history recorded as (yes/no) and Provisional restoration at the time of implant placement recorded as (yes/no).
- Procedural variables for ARP: including type of bone graft used recorded as (Allograft/Xenograft); Type of barrier used recorded (Collagen Plug, Bioresorbable Membrane or Non-resorbable Membrane): Flapped or flapless procedure; Loss of walls of the socket (yes/no).

- Procedural variables for IP: included type of bone graft used recorded as (Autograft/Allograft/Xenograft); location of bone graft placed (buccal/lingual/mesial/distal); one-stage or two-stage; implant diameter (wide/narrow) and length (long/short); and time from ARP to IP.

- Prosthetic variables: included type of the final restoration (screw retained/cement retained) and design of the final restoration (single tooth/fixed partial denture).

\section{STATISTICAL ANALYSIS}

Summary statistics were expressed for the demographic variables as frequencies and percentages. Generalized Estimating Equations (GEE) methodology was used to fit logistic regression model and to estimate the ARP effects univariately. The correlation among the treatment outcomes within one patient was also accounted for using GEE. A 10\% change in the odds ratio for logistic models was used to determine the confounders with ARP effect for the outcomes. Final ARP estimates were reported with adjusting the confounders. A 2-sided 0.05 alpha level was used to define statistical significance. SAS 9.4 was used for all the analyses.

\section{RESULTS \\ Patient sample}

Out of a total 1640 charts reviewed, 829 charts (1416 sites) were included based on the inclusion and exclusion criteria. Six hundred sixty-two charts were excluded due to lack of adequate information, 137 charts due to immediate implant placement, 10 charts due to missing records and two charts for using biologic agents with the extraction procedure. Thus, the final sample (829 patients) included 367 males (44.3\%) and 462 females (55.7), mean age 65.8 years, range from 24 to 93 years (Table 1). Ninety-three $(12.74 \%)$ patients reported being smokers, $96(11.69 \%)$ patients were diabetic and $52(6.33 \%)$ patients reported receiving treatment that affects bone metabolism. 1170 implants were included in ARP group and 246 implants were included in the Naturally Healing $(\mathrm{NH})$ group (Figure 1). Twenty-six implants $(2.22 \%)$ failed in ARP group and 11 implants $(4.47 \%)$ failed in the $\mathrm{NH}$ group. 
Table-1: Demographic Data for Included Subjects

\begin{tabular}{|l|l|}
\hline Characteristics & Mean (SD) or Count (\%) \\
\hline Age (years) & $65.8(11.5)$ \\
\hline Gender & \\
\hline Female & $462(55.8)$ \\
\hline Male & $366(44.2)$ \\
\hline Diabetes Mellitus & $96(11.69)$ \\
\hline Smoking & $93(12.74)$ \\
\hline History of Periodontal Disease & $257(35.16)$ \\
\hline Type of Operator & \\
\hline Specialist & $370(44.63)$ \\
\hline General Dentists & $459(53.37)$ \\
\hline Operator experience & \\
\hline Faculty & $123(14.84)$ \\
\hline Resident & $706(85.16)$ \\
\hline
\end{tabular}

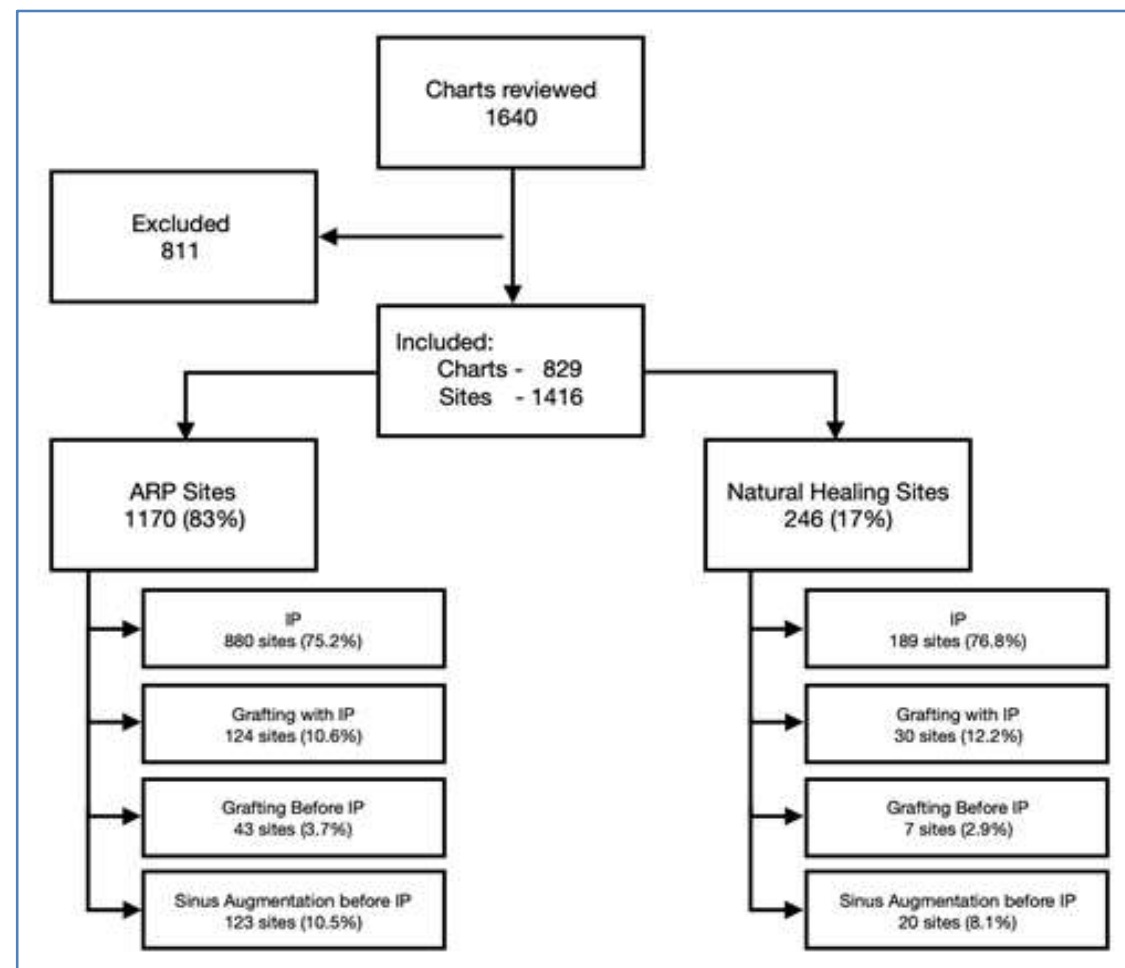

Fig-1: Initial Distribution and Selection of Records. ARP = Alveolar Ridge Preservation, IP = Implant Placement .

\section{Site Distribution}

The distribution of sites receiving the implant placement is shown in Table 2. The posterior segments were more frequently subject to ridge preservation and implant placement than anterior sites. In the incisor region, 196 maxillary sites compared with 48 mandibular sites received implants. In the canine region, 110 maxillary sites compared with 77 mandibular sites received implants. Three hundred fifty-four implants were placed in the maxillary premolar region and 172 implants were placed in the mandible. In the molar region, 179 maxillary sites compared with 280 mandibular sites received implants. Most of the extractions were due to periodontal disease, endodontic pathology and non-restorable tooth (Table 2).

Table-2: Site Distribution

\begin{tabular}{|l|l|l|l|l|l|l|l|l|}
\hline & \multicolumn{2}{|l|}{ Tooth type } \\
\cline { 2 - 9 } & $\begin{array}{l}\text { Central } \\
\text { Incisors }\end{array}$ & $\begin{array}{l}\text { Lateral } \\
\text { Incisors }\end{array}$ & Canine & $\begin{array}{l}\text { First } \\
\text { Premolar }\end{array}$ & $\begin{array}{l}\text { Second } \\
\text { Premolar }\end{array}$ & $\begin{array}{l}\text { First } \\
\text { Molar }\end{array}$ & $\begin{array}{l}\text { Second } \\
\text { Molar }\end{array}$ & $\begin{array}{l}\text { Third } \\
\text { Molar }\end{array}$ \\
\hline Maxillary & 95 & 101 & 110 & 180 & 174 & 153 & 26 &. \\
\hline Mandibular & 11 & 37 & 77 & 73 & 99 & 214 & 65 & 1 \\
\hline
\end{tabular}


F Felemban et al., Saudi J Oral Dent Res, August, 2020; 5(8): 417-424

\section{Biomaterials}

The ridge preservation protocol included application of particulate allogenic or xenogenic bone substitute material covered using a collagen plug or barrier membrane (resorbable or non-resorbable). Grafting at the time of implant placement protocol included application of autogenous, allogenic and xenogeneic bone graft.

\section{Outcome}

Univariate analysis showed that the ARP procedure was not associated with any of the three outcomes: having additional grafting at time of IP $(\mathrm{p}=0.3985)$, grafting before IP $(\mathrm{p}=0.4959)$ and sinus augmentation $\quad(p=0.3242) \quad$ (Table 3$)$. Table 4 summarized the multivariate analysis with adjusting the confounder for ARP. The type of the operator was confounded ARP effect for the need for grafting at IP. Smoking, the use of membrane and loss of socket wall confounded the ARP effect for the need for grafting before IP. History of periodontal disease confounded ARP effect for the need for sinus augmentation measures. Although the odds ratios of ARP group changed more than $10 \%$ compared to the unadjusted univariate ARP model, these confounders didn't alter the statistical inference of ARP effect. The ARP procedure was still not significantly associated with the outcomes.

Table-3: Univariate Analyses with ARP Effect

\begin{tabular}{|l|l|c|c|c|}
\hline \multicolumn{1}{|c|}{ Outcomes } & Characteristics & Odds ratio & 95\% CI & p-value \\
\hline Need grafting at IP & Had ARP (ref=No ARP) & 0.83 & $0.54,1.28$ & 0.3985 \\
\hline Need grafting before IP & Had ARP (ref=No ARP) & 1.33 & $0.58,3.06$ & 0.4959 \\
\hline Need for sinus augmentation & Had ARP (ref=No ARP) & 1.33 & $0.76,2.33$ & 0.3242 \\
\hline
\end{tabular}

$A R P=$ Alveolar Ridge Preservation, $I P=$ Implant Placement

Table-4: Multivariate Analyses Adjusting for the Confounders

\begin{tabular}{|l|l|l|l|r|}
\hline Outcomes & Characteristics & Odds ratio & 95\% CI & p-value \\
\hline Need grafting at IP & Had ARP (ref=no ARP) & 0.94 & $0.61,1.45$ & 0.7679 \\
\hline & Periodontist (ref=General dentist) & 2.18 & $1.54,3.08$ & $<.0001$ \\
\hline Need grafting before IP & Had ARP (ref=no ARP) & 1.2 & $0.48,3.04$ & 0.6932 \\
\hline & Smoking (ref=No) & 1.16 & $0.48,2.76$ & 0.7455 \\
\hline & Membrane (ref=No membrane) & 2.1 & $1.02,4.43$ & 0.0447 \\
\hline & Missing wall (ref=No missing wall) & 2.06 & $0.9,4.69$ & 0.0864 \\
\hline Need for sinus augmentation & Had ARP (ref=no ARP) & 1.17 & $0.64,2.13$ & 0.6073 \\
\hline & Periodontal disease (ref=No) & 0.88 & $0.56,1.38$ & 0.5731 \\
\hline
\end{tabular}

$A R P=$ Alveolar Ridge Preservation, $I P=$ Implant Placement

Table-5: Significant Characteristics from Separate Models Adjusting for ARP Effect

\begin{tabular}{|l|l|r|r|r|r|}
\hline Outcomes & Characteristics & $\begin{array}{r}\text { Odds } \\
\text { ratio }\end{array}$ & $\begin{array}{r}\text { p5\% CI } \\
\text { value }\end{array}$ \\
\hline Need grafting at IP & Gender & Male(ref=Female) & 0.57 & $0.4,0.81$ & 0.0018 \\
\hline & Operator type & Periodontist (ref=General dentist) & 2.18 & $1.54,3.08$ & $<.0001$ \\
\hline & Implant diameter & Narrow (ref=Regular) & 3.18 & $2.12,4.76$ & $<.0001$ \\
\hline Need grafting before IP & Use of membrane & Membrane (ref=No membrane) & 2.6 & $1.38,4.89$ & 0.003 \\
\hline & $\begin{array}{l}\text { Loss of socket } \\
\text { wall }\end{array}$ & Missing wall (ref=No missing & 2.89 & $1.44,5.83$ & 0.003 \\
& wall) & & & \\
\hline & Implant diameter & Narrow (ref=Regular) & 2.57 & $1.29,5.12$ & 0.0073 \\
\hline & Implant length & Short (ref=Regular) & 2.19 & $1.01,4.74$ & 0.0467 \\
\hline Need for sinus augmentation & Operator type & Periodontist (ref=General dentist) & 1.48 & $1.01,2.17$ & 0.0424 \\
\hline & Implant diameter & Narrow (ref=Regular) & 0.04 & $0.01,0.32$ & 0.002 \\
\hline
\end{tabular}

$A R P=$ Alveolar Ridge Preservation, IP = Implant Placement, Implant diameter; Narrow $\leq 3.3 \mathrm{~mm}$, Regular $>3.3 \mathrm{~mm}$, Implant length; Short $\leq 8 m m$, Regular $>$

Upon considering each potential confounding factor with ARP effect separately. There were some characteristics are associated with the outcomes univariatly when we adjusted ARP effect (Table 5). In the models adjusted for ARP, males have a lower odd of needing for grafting at IP compare to females $(\mathrm{OR}=0.57,95 \% \mathrm{CI}: 0.4,0.81)$. The odds of needing for grafting at IP are higher with periodontist $(\mathrm{OR}=2.18$, $95 \%$ CI: $1.54,3.08)$ and using narrow implant
(OR=3.18, 95\% CI: 2.12, 4.76). The odds of needing for grafting before IP are higher in the groups with using of membrane ( $\mathrm{OR}=2.6,95 \%$ CI: $1.38,4.89)$, losing of socket wall (OR=2.89, 95\% CI: $1.44,5.83)$, using narrow implant $(\mathrm{OR}=2.57,95 \% \mathrm{CI}: 1.29,5.12)$ and using short implant (OR=2.19, 95\% CI: 1.01, 4.74). The odds of needing for sinus augmentation is higher with the periodontist (OR=1.48, $95 \% \mathrm{CI}: 1.01,2.17)$ but 
this odd is lower for using the narrow implant $(\mathrm{OR}=0.04,95 \% \mathrm{CI}: 0.01,0.32)$.

\section{DISCUSSION}

The primary aim of this retrospective study is to evaluate the influence of ARP procedure on the future IP outcome. In addition to identifying factors associated with more need to graft prior or at IP procedure. The results of this study showed that there is weak association between ARP procedure and reducing the need for grafting at IP, grafting prior to IP and sinus augmentation.

\section{Grafting at Implant placement}

This study failed to show any association between ARP and grafting at the time of IP. Type of operator were found to be a cofounder due to $10 \%$ change in the odds ratio between the univariate and multivariate analyses. Although, this cofounder did not change the ARP effect. Female patient, sites treated by periodontist or received narrow implant diameter showed statistically significant association with more need for grafting at IP irrespective to ARP procedure. There is not clear explanation and justification why female needed more grafting at IP. In regard the sites treated by specialist having a higher OR, it could be associated with the greater level of the comfort and confidence to graft rather than placing the implant in non-ideal position or placing a smaller size implant. However for the sites received narrow size implant were associated with more need for grafting at IP might be due to having a compromised implant site.

\section{Grafting prior to implant placement}

There was no evident association in the analysis between ARP procedure and grafting prior to implant. Upon adjusting for cofounder, smoking, missing socket wall and following extraction and the use of barrier membrane with ARP showed 10\% change in the odds ratio. Regardless to ARP, the use of membrane, missing socket wall, narrow and short implants had a greater association with grafting prior to IP. It has been reported that the use of membrane with ARP procedure had a beneficial effect on preservation of mid-buccal and mid-lingual bone height [23]. This led to the assumption that by using a membrane resulted in better ridge maintenance, which would reduce the need to subsequent grafting before or at the time of IP. However, this study showed that sites that received a membrane at the time of ARP showed more need for grafting at the time of IP compared to the sites receiving ARP without a membrane. This finding could be due to the use of the membrane is limited for compromised sites, such as a missing buccal wall or the presence of a fenestration. Similarly, one way to avoid extensive grafting in compromised site in to place narrow diameter implant.

\section{Sinus augmentation}

The finding was similar to the pervious outcome, were no statistically significant association with alveolar ridge preservation. History of periodontal disease was found to be a confounded variable; however, it was not associated with more need for sinus augmentation. Type of operator and wide implant diameter were more associated with higher need of sinus augmentation.

In a randomized control trial, Barone et al. [16] evaluated and compared changes of hard and soft tissues in post-extraction sockets which received ARP procedure using xenograft and a collagen membrane, with extraction only in 58 subjects [36]. ARP group showed an average of $1.6 \mathrm{~mm}$ horizontal ridge reduction, while extraction group had $3.6 \mathrm{~mm}$ horizontal ridge reduction. Seven percent of the ARP group required further grafting at the time of IP, while extraction group need grafting $42 \%$ of the sites. This significant difference could be associated with the traumatic extraction procedure for extraction group compared to the extraction of the ARP group. The finding of this study disagree with the results of our study, were no significant difference was found between the 2 groups. More recently [32] reported dimensional difference in radiographic study utilizing cone beam computed tomography comparing ARP using Freeze Dried Bone Allograft (FDBA) in addition non resorbable dense polytetrafluoroethylene (d-PTFE) membrane in the molar region [32]. The difference in horizontal ridge reduction was $1.48 \mathrm{~mm}$ in the favor of the ARP. Bone grafting at time of IP was required in $25 \%$ of implants in the group with no ARP versus $10 \%$ of implants in the ARP group. The results of this investigation refute the finding of the previous studies and agree with the finding of ours study. Additional bone grafting procedure at time of IP was needed $19.79 \%$ in ARP group and $18.85 \%$ in no ARP group.

This retrospective study does not indicate that ARP does not have a beneficial effect. ARP procedure has a significant effect on reducing the volumetric changes after the extraction [23]. The bigger question is if this addition alveolar ridge dimension would have a significant impact on the future implant placement [27]. The previous question was asked in the EAO $4^{\text {th }}$ workshop [26]. There was no difference in all evaluated outcomes except for the need of additional grafting at the time of IP. The need for grafting at the time of implant placement was not described clearly in 4 out of the 6 included studies $[33,19,34,35]$. One study reported that more traumatic extraction has been performed for the sites that did not receive ARP [36]. From the results of this systematic review, the effect of ARP on the subsequent IP still not clear. 
This is the first retrospective study which evaluated the influence of ARP on the implant related outcome. This study included large sample of 829 patients (1416 implants) in the analysis, which will make the hypothesis test more sensitive. Multiple different patient, site and surgical related variables were considered in the analysis.

There are several limitations to this study: retrospective designs which add the risk of selection and observation bias. Furthermore, inability to place implant due to site deficiency was not considered in the study. The drawback for trying to identify the patients who received extractions then evaluating if implant was placed is that there are multiple reasons for not placing implant (i.e. financial, patient preference, patient moved, etc.). In addition to that some of the implant placed by a relative inexperienced resident, however different level of experienced operator was included. No association was found between implant placed by faculty, resident and implant related outcomes.

In conclusion, this retrospective study showed a weak association between alveolar ridge preservation and the addition need for future grafting at the time of implant placement, grafting prior to implant placement and sinus augmentation. No conclusion should be drawn from the strong association between the use of a membrane with alveolar ridge preservation in comparison to alveolar ridge preservation with a collagen plug, and the use of non-resorbable membrane verses resorbable. Large sample size randomized control trials with a standardized treatment protocol are needed to evaluate the effect of ARP on the future implant related outcomes. However, careful evaluation of the demographic, site, and procedural related factors are needed.

\section{REFERENCES}

1. Cohen, R., Cochran, D.L., Blieden, T., \& Fine, J. (2001). Glossary of periodontal terms (American Academy of Periodontology).

2. Lekholm, U., \& Zarb, G.A. (1985). Patient selection and preparation. In: Brånemark, P.- I., Zarb, G.A., Albrektsson, T., eds. Tissue Integrated Prostheses: Osseointegration in Clinical Dentistry (Chicago: Quintessence Publ Co.).

3. Pietrokovski, J., \& Massler, M. (1967). Alveolar ridge resorption following tooth extraction. $J$. Prosthet. Dent. 17, 21-27.

4. Schropp, L., Wenzel, A., Kostopoulos, L., \& Karring, T. (2003). Bone healing and soft tissue contour changes following single-tooth extraction: a clinical and radiographic 12-month prospective study. Int. J. Periodontics Restorative Dent. 23, 313-323.

5. Araújo, M.G., and Lindhe, J. (2005). Dimensional ridge alterations following tooth extraction. An experimental study in the dog. J. Clin. Periodontol, 32, 212-218.

6. Van der Weijden, F., Dell'Acqua, F., and Slot, D.E. (2009). Alveolar bone dimensional changes of post-extraction sockets in humans: a systematic review. J. Clin. Periodontol, 36, 1048-1058.

7. Chappuis, V., Engel, O., Reyes, M., Shahim, K., Nolte, L.-P., and Buser, D. (2013). Ridge Alterations Post-extraction in the Esthetic Zone. $J$. Dent. Res. 92, 195S-201S.

8. Chappuis, V., Engel, O., Shahim, K., Reyes, M., Katsaros, C., and Buser, D. (2015). Soft Tissue Alterations in Esthetic Postextraction Sites: A 3Dimensional Analysis. J. Dent. Res. 94, 187S-93S.

9. Farmer, M., \& Darby, I. (2014). Ridge dimensional changes following single-tooth extraction in the aesthetic zone. Clin. Oral Implants Res, 25, 272 277.

10. Buser, D., Martin, W., \& Belser, U.C. (2004). Optimizing esthetics for implant restorations in the anterior maxilla: anatomic and surgical considerations. Int. J. Oral Maxillofac. Implants, 19 Suppl, 43-61.

11. Evans, C.D.J., \& Chen, S.T. (2008). Esthetic outcomes of immediate implant placements. Clin. Oral Implants Res. 19, 73-80.

12. Ashman, A., \& Bruins, P. (1982). A new immediate hard tissue replacement (HTR)TM for bone in the oral cavity. J. Oral Implantol. 10, 419452.

13. Ashman, A., and Bruins, P. (1985). Prevention of alveolar bone loss postextraction with HTR grafting material. Oral Surg. Oral Med. Oral Pathol, 60, 146-153.

14. Aimetti, M., Romano, F., Griga, F.B., and Godio, L. (2009). Clinical and histologic healing of human extraction sockets filled with calcium sulfate. Int. $J$. Oral Maxillofac. Implants, 24, 902-909.

15. Barone, A., Aldini, N.N., Fini, M., Giardino, R., Calvo Guirado, J.L., \& Covani, U. (2008). Xenograft Versus Extraction Alone for Ridge Preservation After Tooth Removal: A Clinical and Histomorphometric Study. J. Periodontol, 79, 1370-1377.

16. Barone, A., Orlando, B., Cingano, L., Marconcini, S., Derchi, G., \& Covani, U. (2012). A Randomized Clinical Trial to Evaluate and Compare Implants Placed in Augmented Versus Non-Augmented Extraction Sockets: 3-Year Results. J. Periodontol, 83, 836-846.

17. Camargo, P. M., Lekovic, V., Weinlaender, M., Klokkevold, P. R., Kenney, E. B., Dimitrijevic, B., ... \& Orsini, M. (2000). Influence of bioactive glass on changes in alveolar process dimensions after exodontia. Oral Surgery, Oral Medicine, Oral Pathology, Oral Radiology, and Endodontology, 90(5), 581-586.

18. Festa, V.M., Addabbo, F., Laino, L., Femiano, F., \& Rullo, R. (2013). Porcine-Derived Xenograft Combined with a Soft Cortical Membrane versus 
Extraction Alone for Implant Site Development: A Clinical Study in Humans. Clin. Implant Dent. Relat. Res. 15, 707-713.

19. Iasella, J.M., Greenwell, H., Miller, R.L., Hill, M., Drisko, C., Bohra, A.A., \& Scheetz, J.P. (2003). Ridge Preservation with Freeze-Dried Bone Allograft and a Collagen Membrane Compared to Extraction Alone for Implant Site Development: A Clinical and Histologic Study in Humans. $J$. Periodontol, 74, 990-999.

20. Atieh, M. A., Alsabeeha, N. H., Payne, A. G., Duncan, W., Faggion, C. M., \& Esposito, M. (2015). Interventions for replacing missing teeth: alveolar ridge preservation techniques for dental implant site development. Cochrane Database of Systematic Reviews, (5).

21. Horváth, A., Mardas, N., Mezzomo, L.A., Needleman, I.G., \& Donos, N. (2013). Alveolar ridge preservation. A systematic review. Clin. Oral Investig, 17, 341-363.

22. MacBeth, N., Trullenque-Eriksson, A., Donos, N., and Mardas, N. (2017). Hard and soft tissue changes following alveolar ridge preservation: a systematic review. Clin. Oral Implants Res, 28, 982-1004.

23. Avila-Ortiz, G., Elangovan, S., Kramer, K.W.O., Blanchette, D., \& Dawson, D.V. (2014). Effect of Alveolar Ridge Preservation after Tooth Extraction: A Systematic Review and Metaanalysis. J. Dent. Res, 93, 950-958.

24. Chan, H. L., Lin, G. H., Fu, J. H., \& Wang, H. L. (2013). Alterations in bone quality after socket preservation with grafting materials: a systematic review. International Journal of Oral \& Maxillofacial Implants, 28(3).

25. De Risi, V., Clementini, M., Vittorini, G., Mannocci, A., \& De Sanctis, M. (2015). Alveolar ridge preservation techniques: a systematic review and meta-analysis of histological and histomorphometrical data. Clin. Oral Implants Res. $26,50-68$.

26. Mardas, N., Trullenque- Eriksson, A., MacBeth, N., Petrie, A., \& Donos, N. (2015). Does ridge preservation following tooth extraction improve implant treatment outcomes: a systematic review: Group 4: Therapeutic concepts \& methods. Clinical oral implants research, 26, 180-201.

27. Sanz, M., Donos, N., Alcoforado, G., Balmer, M., Gurzawska, K., Mardas, N., \& Thoma, D. S. (2015). Therapeutic concepts and methods for improving dental implant outcomes. Summary and consensus statements. The 4th EAO Consensus Conference 2015. Clinical Oral Implants Research, 26, 202-206.
28. Willenbacher, M., Al- Nawas, B., Berres, M., Kämmerer, P. W., \& Schiegnitz, E. (2016). The effects of alveolar ridge preservation: a metaanalysis. Clinical implant dentistry and related research, 18(6), 1248-1268.

29. Armitage, G. C. (1999). Development of a classification system for periodontal diseases and conditions. Annals of periodontology, 4(1), 1-6.

30. Ortega- Oller, I., Suárez, F., Galindo- Moreno, P., Torrecillas- Martínez, L., Monje, A., Catena, A., \& Wang, H. L. (2014). The influence of implant diameter on its survival: a meta- analysis based on prospective clinical trials. Journal of periodontology, 85(4), 569-580.

31. Renouard, F., \& Nisand, D. (2006). Impact of implant length and diameter on survival rates. Clinical oral implants research, 17(S2), 3551.

32. Walker, C. J., Prihoda, T. J., Mealey, B. L., Lasho, D. J., Noujeim, M., \& Huynh- Ba, G. (2017). Evaluation of healing at molar extraction sites with and without ridge preservation: A randomized controlled clinical trial. Journal of periodontology, 88(3), 241-249.

33. Cardaropoli, D., Tamagnone, L., Roffredo, A., \& Gaveglio, L. (2014). Relationship Between the Buccal Bone Plate Thickness and the Healing of Postextraction Sockets With/Without Ridge Preservation. International Journal of Periodontics \& Restorative Dentistry, 34(2).

34. Sisti, A., Canullo, L., Mottola, M. P., Covani, U., Barone, A., \& Botticelli, D. (2012). Clinical evaluation of a ridge augmentation procedure for the severely resorbed alveolar socket: multicenter randomized controlled trial, preliminary results. Clinical oral implants research, 23(5), 526535.

35. Spinato, S., Galindo- Moreno, P., Zaffe, D., Bernardello, F., \& Soardi, C. M. (2014). Is socket healing conditioned by buccal plate thickness? A clinical and histologic study 4 months after mineralized human bone allografting. Clinical Oral Implants Research, 25(2), e120-e126.

36. Barone, A., Ricci, M., Tonelli, P., Santini, S., \& Covani, U. (2013). Tissue changes of extraction sockets in humans: a comparison of spontaneous healing vs. ridge preservation with secondary soft tissue healing. Clinical oral implants research, 24(11), 1231-1237.

37. Cardaropoli, D., Roffredo, A., \& Cardaropoli, G. (2012). Socket preservation using bovine bone mineral and collagen membrane: a randomized controlled clinical trial with histologic analysis. International Journal of Periodontics \& Restorative Dentistry, 32(4). 